\title{
Utility of telemedicine in the COVID-19 era
}

\author{
Gates B. Colbert ${ }^{1, *}$, A. Verner Venegas-Vera ${ }^{2}$ and Edgar V. Lerma ${ }^{3}$ \\ ${ }^{1}$ Assistant Clinical Professor, Division of Nephrology, Texas A\&M College of Medicine at Dallas, 75246, USA \\ ${ }^{2}$ Nephrologist, Division of Internal Medicine, Mexican Institute of Social Security, Merida, Yucatan, 97150, Mexico \\ ${ }^{3}$ Clinical Professor of Medicine, University of Illinois at Chicago/Advocate Christ Medical Center, Oak Lawn, 60453, IL, USA \\ *Correspondence: Gates.Colbert@BSWHealth.org (Gates B. Colbert)
}

DOI: $10.31083 /$ i.rcm.2020.04.188

This is an open access article under the CC BY 4.0 license (https://creativecommons.org/licenses/by/4.0/).

Previously it has been demonstrated that telehealth (TH) could help cover the gaps in health attention in remote locations. Today the expanded capabilities have transformed TH delivery, and from the beginning of the coronavirus pandemic, it has remained one of our biggest allies. Telehealth has become a central piece in patient healthcare delivery during COVID-19 pandemic era. Telehealth allows health care services to reach patients in their homes, keeping other patients safe through social distancing and maintaining self-quarantine. Within this administration of health, $\mathrm{TH}$ allows health care providers to focus more resources to pandemic usage and at the same time continue caring for the health of non COVID-19 patients. During this time, clinicians are expanding knowledge about TH capabilities, such as application of forward triage as a tool to avoid patient contact in emergency departments. While previously $\mathrm{TH}$ was mainly used for primary care needs, specialized and urgent care health is now being utilized more than ever before. These advantages comes with limitations, some of them include a limited physical exam, lack of access to diagnostic testing or imaging, and many other piffalls and persistent unmet needs. The 2020 pandemic has led to significant improvements leading into the next generation of telemedicine.

\section{Keywords}

Telemedicine; telehealth; COVID-19; healthcare

\section{Introduction}

Telemedicine is a form of caring for patients using technology as a means of healthcare communication and delivery. This application of care has been around for decades and is as simplistic as a patient calling his or her physician for advice and treatment. In today's world of advancing technology, including live video and text messaging, telehealth has quickly and broadly expanded its scope and capabilities. Prior to the coronavirus disease 2019 (COVID-19) pandemic in the United States, thousands of physicians and health care professionals $(\mathrm{HCP})$ provided care to patients in some way with telemedicine. Previous work has already described the role of telehealth importance during disasters and public health emergencies (Lurie and Carr, 2018). With the novel COVID-19 crisis erupting in 2020, and the high uncertainty of spread and infectivity, the urgency for telemedicine quickly became an important way to manage patients' healthcare while keeping them safe through social distancing and maintaining self-quarantine (Cervino et al., 2020).

In April 2020, healthcare systems in the United States, through the urging of the Centers for Disease Control, encouraged all outpatient clinics, hospitals and ambulatory surgical centers, to limit all non-essential activity. This was implemented with the goal of limiting COVID-19 spread and prevention of personal protective equipment (PPE) depletion (CDC, 2020; Centers for Medicare and Medicaid Services, 2020). As patients were encouraged to follow stay-at-home orders and clinics were closed to inperson visits, telemedicine quickly became a necessary component for non-emergency healthcare. Synchronous telehealth was enacted, where a HCP and patient interact in real time, as a primary means of healthcare for many clinics and healthcare systems (Koraishy and Rohatgi, 2020). As physicians and HCPs were instructed to use this form of healthcare delivery, several outcomes were quickly visible on a global scale (Bashshur et al., 2020). Patients had good outcomes and satisfaction with outpatient follow up and nonurgent condition consultations. Hardware infrastructure with widespread connected devices, dubbed the internet of things (IoT) e.g. tablets, phones, and watches, allowed direct point of care delivery (Ting et al., 2020). Electronic health records could be maintained by the HCP with diagnostic testing, medication orders, and follow up appointments available by digital creation. As well, the ability for patients to practice physical distancing and self-quarantine were enhanced with decreased needs to present to a crowded waiting room (Bashshur et al., 2020).

Telemedicine consultations require the same amount of rigorous investigation and astute attention to accurately meet the standard of care. Several components are necessary to complete a meaningful visit for patient treatment. Comprehensive documentation is key to a successful legal, billable, and Health Insurance Portability and Accountability Act (HIPAA) compliant encounter. The assessment, diagnosis, and treatment plan should be well documented to support the HCP plan and any medications or tests that are needed. If a HCP orders a test or imaging, the HCP is responsible for following up on the findings and determining the next steps of care and assumes sole liability. Correct Current Procedu- 
ral Terminology (CPT) diagnosis codes must be documented in the encounter as well. Clinicians will be very familiar with these necessary elements to a patient encounter, as they exist in the current standard of care for live physical consultations.

\section{Previous Telehealth Expereience}

Patients prior to the pandemic were increasingly using telehealth and smart technology in monitoring their health on a daily or hourly basis. Several reports and studies have demonstrated the success of using telemedicine in managing chronic conditions (Fig. 1), such as Nephrology clinics, while adhering to the techniques suggested above (Lea and Tannenbaum, 2020). In fact, Canadian physicians have been using telemedicine to care for remote patients on dialysis since the 1980s (Bernstein et al., 2010). Another example of telemedicine details the management of kidney disease and dialysis treatments by HCPs to isolated Pacific Islands patients (Michel et al., 2020). A more recent study using a smartphone app in patients with chronic kidney disease showed reductions in systolic and diastolic blood pressure with smartphone intervention (Singh et al., 2019). Many HCPs and Cardiologists are familiar with the Apple Watch heart rate capabilities and KardiaMobile rhythm detection availability, both providing meaningful data that can shape healthcare outcomes ( $\mathrm{Li}$ et al., 2019). Published cases are now rapidly being reported in the literature describing how different specialties across the world used telemedicine to care for patients during COIVD-19 pandemic lock down periods. An Italian cardiology group described how to manage heart failure symptoms using telemedicine through phone calls, video conferencing, and email exchanges to keep patients on goal directed therapy when in person consultation was not available (Salzano et al., 2020). Up to 58\% of patients who participated in the study accessed telemedicine services, with $51 \%$ of those patients having a clinical decision adjustment performed. No patients in the Italian 103-person study contracted COVID-19 during the three-month period. Patients' choosing telemedicine over in-person care have become visible almost immediately at the beginning of the COVID-19 pandemic. It has been reported in Italy and elsewhere, a decrease in ED presentations for acute coronary symptoms during the early pandemic months. As patients were fearful to go to the ED, a significant decrease occurred in patients seeking in-person care compared to previous time periods (De Filippo et al., 2020; Piccolo et al., 2020).

\section{Emergency Use of Telehealth}

The use of Emergency services must remain available to patients at all times in a modern society. Strategies to triage patients as they enter these services is needed to maintain order in providing emergency care to the sickest quickly. "Forward triage" is a process that sorts these patients before they arrive in the emergency department (Cervino and Oteri, 2020). Telemedicine is a way to help this process and allows patients to be screened before entering the doors of the hospital, potentially exposing themselves and families to contract illness such as COVID-19. Currently over 50 American health systems already have telemedicine triage programs in place and aggressively used the opportunity to initially care for patients virtually (Hollander and Carr, 2020). Outsourcing these services is a way for smaller and less resource rich systems to care for their patients in order to limit cost and resource utilization. Teladoc Health and American Well both are public telehealth companies that played a role to screen patient's symptoms and provide care before referral to an in-person hospital consultation. Many health systems, such as Jefferson Health in Pennsylvania, used telemedicine to screen patients who were experiencing symptoms of COVID-19 (Hollander and Carr, 2020). Patients already in the system with outpatient established care, were asked to use telemedicine for direct virtual evaluation to determine if testing was necessary. This prevented patients from exposing themselves to other patients without a centralized location, and allowed the clinicians to treat patients without COVID-19 suspicion at home for monitoring.

\section{Critical Care Use of Telehealth}

Inpatient telemedicine services are increasingly being used to care for COVID-19 positive patients admitted to ward units or intensive care units (ICU). Electronic intensive care unit (e-ICU) monitoring programs allow clinicians and nursing staff to monitor and communicate with patients remotely (Hollander and Carr, 2020). This allows for two-way communication, vital status monitoring, well-being of the patient, direct video of a patient's ventilator, and even dialysis treatment machines. With limited direct patient contact, exposure for virus spread is limited from patient to caregiver, and caregivers' virus spread to naïve patients in the hospital. Tele-critical care (TCC) has been pushed to the forefront with COVID-19 positive patients filling up intensive care beds during the first half of 2020 (Singh et al., 2020). Several health systems implemented operational plans to set up ICU rooms with video and audio capabilities for physicians, nursing, and providers to care for patients remotely. To make this model work, teamwork is essential with live communication and feedback highly beneficial. In order to implement these forms of care, heavy investment financially in equipment and training is necessary to perform at the same stand of care as in person.

\section{Benefits of Telehealth}

There are many benefits to telemedicine for both the patient and the HCP outside of a pandemic emergency time frame. The patient has access to a physician or HCP wherever he or she is located and at unpredicted time periods. The patient may live in a rural area with few HCPs, or have a strict schedule and that doesn't allow time to efficiently attend an office visit. There is also less expense for the patient with no travel costs, less time off of work, and potentially lower childcare costs. The encounter may be more private as a patient does not need to be in a waiting room or large hospital setting. As well, the risk of communicable infections is reduced as the patient will have limited exposures. The HCP also benefits from an encounter of telemedicine. Training can be initiated and completed through online modules using the same software that will be used with actual patient encounters. The timing may be more flexible for the HCP to begin and finish their treatment session. Increased efficiency exists without waiting on patients to arrive or rescheduling missed appointments and cancellations. The HCP may be able to take care of patients outside their usual geography or office settings. To the benefit of patient care and promotion of telemedicine spread, the Centers for Medicare and 
Dr. Hugo Gernsback's "Teledactyl" 2 Fantasy

A tool with robotic fingers and a projected video feed to examine the patient from afar.

\section{NASA takes on telemedicine. \\ 4 \\ Space Age pushed money into \\ telemedicine field for astronaut's health}

\begin{tabular}{l|l|}
\hline First telehealth system & 6 \\
From Boston Logan Airport to \\
Massachusetts General Hospital. \\
\hline $\begin{array}{l}\text { First successfully defibrillated } \\
\text { patient by telephone }\end{array}$ \\
In Jewish Hospital of St. Louis
\end{tabular}

\section{Invention of World Wide Web}

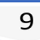

Expanding the capabilities of

telemedicine

"ATA"
The American Telemedicne Association
a non profit organization is created

\begin{tabular}{l|l|} 
"Skype" & 13 \\
Videochat programs and apps like \\
Skype take-off
\end{tabular}

"ARRA"
The American Recovery and
Reinvestment ACT includes health IT
and Telemedicine to stimulate business
in the industry

\section{Mobile Healthcare}

17

Americans use smartphones to research medical information or access health tools

\section{Smartwatch and American Heart}

\section{Association}

Apple Watch Series 4 added an electrical heart sensor approved by FDA
1876
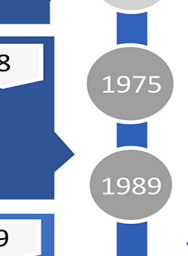

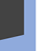

200
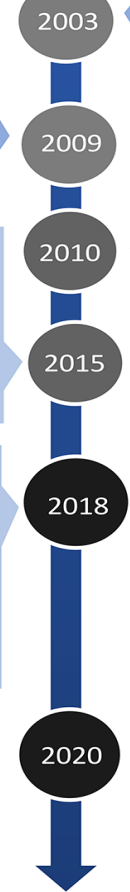

Alexander Graham Bell launches the beginnings of our telecommunications field.

First interactive video link

3

Nebraska psychiatric institute in Omaha and

the Norfolk State Hospital created the first

video link to provide medical care

AT\&T releases "Picturephone" 5

One of the earliest examples of videochat

\section{"STARPAHC" \\ Space Technology Applied to Rural \\ Papago Advanced Health Care}

NASA partners with Indian Health Services

in Papago, Arizona providing health care

through a high tech. equipped van.

First International Telemedicine

Project

NASA launches Space Bridge to Armenia to offer U.S. medical support after a

devastating earthquake

Medicare gets in the Telemedicine

Game

CMS begins paying for telehealth

consultations for patients who live in rural

areas

\section{VA Telehealth}

The U.S. Department of Veterans Affairs, (the largest healthcare system in U.S.) adopted Telehealth and goes nation wide

\section{Cutting down costs}

The decade brings rapid expansion in telemedicine as U.S. looks for ways to improve health care cost

\section{VA mission act}

Removes all geographic and licensing barriers to VA telehealth. Also other measures that aim to expand veterans' access to care

\section{Healthcare in times of Crisis}

The pandemic era of COVID-19 impulse telehealth consultations for patients to prevent spread of the disease. Improving platforms and payment methods.

Fig. 1. Timeline highlighting the history of telemedicine from infancy to massive implementation during the COVID-19 pandemic (Zundel, 1997). 
Medicaid Services greatly expanded payment policy to allow reimbursement for televisits at the same rates as in person care (Hollander and Carr, 2020). This was enacted during the COVID-19 pandemic to facilitate this opportunity for patients and HCPs, but it remains to be seen if this change in payment models will be a temporary emergency order or a permanent statute of healthcare.

\section{Limitiations}

Limitations do exist to providing excellent and medically accurate care with telemedicine. Full data and medical histories may be incomplete if totally relying on a patient's interpretation of their problem. A patient's description of their issue may not be as accurate as an in-person encounter with limitations of non-verbal communication. The physical exam is limited without access to expert clinician evaluation. Quick access to diagnostic testing or imaging may also not be available. Not every patient problem and diagnosis will be able to be determined during a telemedicine visit such as abdominal pain or vision changes. Patients with mental disabilities and those with cognitive impairment may not be candidates for telemedicine given inherent communication limitations (López Reboiro et al., 2020). It is up to the medical judgement and expertise of the HCP to determine when an in-person encounter is required. A recent review on the reliability of 28 smartphone applications to track and manage chronic kidney disease data and symptoms describes many pitfalls and persistent unmet needs (Singh et al., 2019). The liability and safety rests on the shoulders of the HCP, as the patient is looking to an established and educated authority to help with their medical problems. Additional hurdles that need to be overcome include privacy issues, data safety, limited integration of services within health systems, and incorporation with current delivery care models such as pharmacies. Chronic telemonitoring needs to be integrated into the model of care after initial consultations. Ideally patients would be cared for directly in person for subsequent follow up visits or consult. If situations and barriers exist to physical care, monitoring of accurate health data, laboratory values, and vital organ monitoring must be available. While we don't have answers to all of these challenges, solutions will continue to present themselves with ongoing interest and investment to meet the needs of telemedicine into 2021.

\section{Conclusion}

Disasters like the COVID-19 global pandemic produce great strain on healthcare systems, delivery systems, and of course the patients seeking medical care. It is an exciting and revolutionary time for health care via telemedicine. The capabilities to help and treat patients experiencing the COVID-19 virus, as well as routine care, have never been greater and in higher demand. The year 2020 will likely be marked as the time period when the public and governments accepted digital technology use as a part of routine healthcare delivery. The scope and improvement will continue to enable a good clinician to treat effectively through virtual means as we continue to evolve in taking excellent care of our patients.

\section{Authors' contributions}

All authors listed contributed equally to the research, writing, and editing of this manuscript.

\section{Acknowledgments}

The authors would like to acknowledge and thank all of the front line healthcare workers who have risked their lives and their personal health to care for so many during this COVID-19 Pandemic.

\section{Conflict of Interest}

The authors declare no conflicts of interest statement.

Submitted: September 15, 2020

Revised: October 12, 2020

Accepted: October 12, 2020

Published: December 30, 2020

\section{References}

Bashshur, R., Doarn, C. R., Frenk, J. M., Kvedar, J. C. and Woolliscroft, J. O. (2020) Telemedicine and the COVID-19 pandemic, lessons for the future. Telemedicine and E-Health 26, 571-573.

Bernstein, K., Zacharias, J., Blanchard, J. F., Yu, B. N. and Shaw, S. Y. (2010) Model for equitable care and outcomes for remote full care hemodialysis units. Clinical Journal of the American Society of Nephrology 5, 645-651.

CDC (2020) CDC Activities and Initiatives Supporting the COVID19 Response and the President's Plan for Opening America. Accessed: https://www.cdc.gov/coronavirus/2019-ncov/downlo ads/php/cdc-activities-initiatives-for-covid-19-response.pdf.

Cervino, G., Fiorillo, L., Surace, G., Paduano, V., Fiorillo, M. T., De Stefano, R., Laudicella, R., Baldari, S., Gaeta, M. and Cicciù, M. (2020) SARS-CoV-2 Persistence: Data Summary up to Q2 2020. Data 5, 81.

Cervino, G. and Oteri, G. (2020) COVID-19 pandemic and telephone triage before attending medical office: Problem or opportunity? Medicina 56, 250.

De Filippo, O., D’Ascenzo, F., Angelini, F., Bocchino, P. P., Conrotto, F., Saglietto, A., Secco, G. G., Campo, G., Gallone, G., Verardi, R., et al. (2020) Reduced rate of hospital admissions for ACS during Covid19 outbreak in Northern Italy. New England Journal of Medicine 383, 88-89.

Fiorillo, L., Cervino, G., Matarese, M., D’Amico, C., Surace, G., Paduano, V., Fiorillo, M. T., Moschella, A., La Bruna, A., Romano, G. L., Laudicella, R., Baldari, S. and Cicciù, M. (2020) COVID-19 surface persistence: A recent data summary and its importance for medical and dental settings. International Journal of Environmental Research and Public Health 17, 3132.

Hollander, J. E. and Carr, B. G. (2020) Virtually perfect? Telemedicine for Covid-19. New England Journal of Medicine 382, 1679-1681.

Koraishy, F. M. and Rohatgi, R. (2020) Telenephrology: an emerging platform for delivering renal health care. American Journal of Kidney Diseases 76, 417-426.

Lea, J. P. and Tannenbaum, J. (2020) The role of telemedicine in providing nephrology care in rural hospitals. Kidney360 1, 553-556.

Li, K. H. C., White, F. A., Tipoe, T., Liu, T., Wong, M. C., Jesuthasan, A., Baranchuk, A., Tse, G. and Yan, B. P. (2019) The current state of mobile phone apps for monitoring heart rate, heart rate variability, and atrial fibrillation: Narrative review. JMIR Mhealth and Uhealth 7, e11606.

López Reboiro, M. L., Sardiña González, C., Castro-Conde, B. A. and López Castro, J. (2020) Telemedicine Yes, But No Telepathy. Acta Médica Portuguesa 33, 624.

Lurie, N. and Carr, B. G. (2018) The role of telehealth in the medical response to disasters. JAMA Internal Medicine 178, 745.

Michel, L., Baroux, N., Frimat, L. and Quirin, N. (2020) Telenephrology and on-site nephrology: Comparable adequate dialysis care to patients living in remote Pacific Islands. Journal of Telemedicine and Telecare 43, 1357633 X1989668.

Centers for Medicare and Medicaid Services (2020) Non-Emergent, Elective Medical Services, and Treatment Recommendations. Available at: https://www.cms.gov/files/document/cms-non-emergen 
t-elective-medical-recommendations.pdf (Accessed: June 09, 2020).

Piccolo, R., Bruzzese, D., Mauro, C., Aloia, A., Baldi, C., Boccalatte, M., Bottiglieri, G., Briguori, C., Caiazzo, G., Calabrò, P., CappelliBigazzi, M., De Simone, C., Di Lorenzo, E., Golino, P., Monda, V., Perrotta, R., Quaranta, G., Russolillo, E., Scherillo, M., Tesorio, T., Tuccillo, B., Valva, G., Villari, B., Tarantini, G., Varricchio, A. and Esposito, G. (2020) Population trends in rates of percutaneous coronary revascularization for acute coronary syndromes associated with the COVID-19 outbreak. Circulation 141, 2035-2037.

Salzano, A., D'Assante, R., Stagnaro, F. M., Valente, V., Crisci, G., Giardino, F., Arcopinto, M., Bossone, E., Marra, A. M. and Cittadini, A. (2020) Heart failure management during the COVID-19 outbreak in Italy: a telemedicine experience from a heart failure university tertiary referral centre. European Journal of Heart Failure 22, 1048-1050.

Singh, K., Diamantidis, C. J., Ramani, S., Bhavsar, N. A., Mara, P., Warner, J., Rodriguez, J., Wang, T. and Wright-Nunes, J. (2019) Patients' and nephrologists' evaluation of patient-facing smartphone Apps for CKD. Clinical Journal of the American Society of Nephrology 14, 523-529.

Singh, J., Green, M. B., Reif, M. S., Thakkar, N. P., Papali, A. (2020) Telecritical care clinical and operational strategies in response to COVID19. Telemedicine and E-Health [in press].

Ting, D. S. W., Carin, L., Dzau, V. and Wong, T. Y. (2020) Digital technology and COVID-19. Nature Medicine 26, 459-461.

Zundel, K. M. (1997) Telemedicine: history, applications, and impact on librarianship. Bulletin of the Medical Library Association 84, 71-79. 